\title{
One-Pot Selective Functionalization of Polysaccharides with Urea ${ }^{+}$
}

\author{
Vanina A. Guntero ${ }^{1,2}$, Micaela Peralta ${ }^{2}$, Pablo Noriega ${ }^{1}$, María N. Kneeteman ${ }^{1}$ and Cristián A. Ferretti 1,* \\ 1 IQAL (UNL-CONICET), Laboratorio Fester-QUÍMICA ORGANICA (FIQ), \\ Universidad Nacional del Litoral, Santa Fe 3000, Argentina; vguntero@sanfrancisco.utn.edu.ar (V.A.G.); \\ pablo.noriega@outlook.com (P.N.); mkneeteman@fiq.unl.edu.ar (M.N.K.) \\ 2 Grupo Productos Naturales, Universidad Tecnológica Nacional Facultad Regional San Francisco, \\ San Francisco 2400, Córdoba, Argentina; micaelaperalta25@gmail.com \\ * Correspondence: cferretti@fiq.unl.edu.ar; Tel.: +54-34-2457-1164 \\ + Presented at the 24th International Electronic Conference on Synthetic Organic Chemistry, \\ 15 November-15 December 2020; Available online: https://ecsoc-24.sciforum.net/.
}

Citation: Guntero, V.A..; Peralta, M.; Noriega, P.; Kneeteman, M.N.; Ferretti, C.A. One-Pot Selective Functionalization of Polysaccharides with Urea. Chem. Proc. 2021, 3, 74. https://doi.org/10.3390/ecsoc-24-08346

Academic Editors: Julio A. Seijas and M. Pilar Vázquez-Tato

Published: 14 November 2020

Publisher's Note: MDPI stays neutral with regard to jurisdictional claims in published maps and institutional affiliations.

Copyright: $@ 2020$ by the authors. Licensee MDPI, Basel, Switzerland. This article is an open access article distributed under the terms and conditions of the Creative Commons Attribution (CC BY) license (http://creativecommons.org/licenses/by/4.0/).

\begin{abstract}
Natural polysaccharides are polymers composed of monosaccharide units bound together by glycosidic linkages. In their native form, these polymers may not be able to provide all the desired properties for a particular application. Thus, the functionalization of their reactive hydroxyl groups would change their surface properties. In this work, we evaluated the derivatization of $\mathrm{OH}$ groups of maltodextrin polysaccharide with urea. Through a one-pot selective procedure carried out at room temperature for $3 \mathrm{~h}$, urea united at maltodextrin through covalent bonding was obtained. The results show that after functionalization with urea, the $\mathrm{pH}$ and solubility in polar solvents of the new material increased. Derivatization of maltodextrin with urea confers stability, and terminal amide groups on the surface of this material represent a versatile reactive functional group for conjugation with other molecules for potential applications in the development of new materials.
\end{abstract}

Keywords: functionalization; maltodextrin; urea; one-pot synthesis

\section{Introduction}

Natural polysaccharides (NPLSs) are the most important macromolecules in nature with functional diversity, which can be considered promising materials for numerous fields ranging from industrial to biomedical applications [1].

Among biopolymers, natural polysaccharides are the most abundant and naturally available polymers, which have gained great popularity over synthetic polymers because of their diverse functions. In addition, NPLSs are documented to be generally recognized as safe (GRAS) for targeting delivery because of their superior properties, including nontoxicity and non-reactogenicity, easy availability on a large scale and relatively less expensive remarkable biocompatibility, and extraordinary biodegradability. They can be easily modified and processed according to the required designs and structures for further application in several areas [2].

Polysaccharides are carbohydrate polymers consisting of at least 10 monosaccharides linked by glycosidic linkages, which are known to be one of the crucial biomacromolecules in the growth and development of living organisms [3]. NPLSs are commonly functional macromolecular biopolymers isolated from several origins, including plant (i.e., cellulose, pectin), animal (e.g., chitosan, chondroitin), microbial (i.e., xanthan gum, pullulan, dextran), and algal (e.g., alginate) [2]. To improve the functions of polysaccharides obtained, physical, chemical, and biological methods have been widely used to modify their structures. 
It is known that the structural features of polysaccharides, such as degree and steric configuration of substitutions, linkages of monosaccharides and substitutes, and molar mass and its distribution, play a critical role in their physicochemical (e.g., solubility and fluid capability) and bioactive properties. Therefore, the modification of natively bioactive polysaccharides to extend their applications is of high importance. The aims of modification or functionalization include, but are not limited to, the improvement and/or introduction of bioactivity, biocompatibility, control of biodegradability, and manufacturing and shaping for biomedical, pharmaceutical, and food applications [4].

Maltodextrin (MD) is a reducing hydrophilic polysaccharide that has been used extensively in the food industry, beverage products, and pharmaceutical industry (Figure 1) $[5,6]$. Maltodextrins $\left(\left(\mathrm{C}_{6} \mathrm{H}_{10} \mathrm{O}_{5}\right)_{n}\right)$ are nonsweet saccharide polymers that consist of D-glucose units linked primarily by $\alpha(1,4)$-glycosidic bonds and have a dextrose equivalent (DE) of less than 20 [7]. Typically, maltodextrins are obtained from starch polymers by acid hydrolysis, enzymatic hydrolysis, or a combination of the two and are characterized by their dextrose equivalent, being the fraction of hydrolyzed glucoside bonds. Maltodextrins with different DE values have different properties [8].

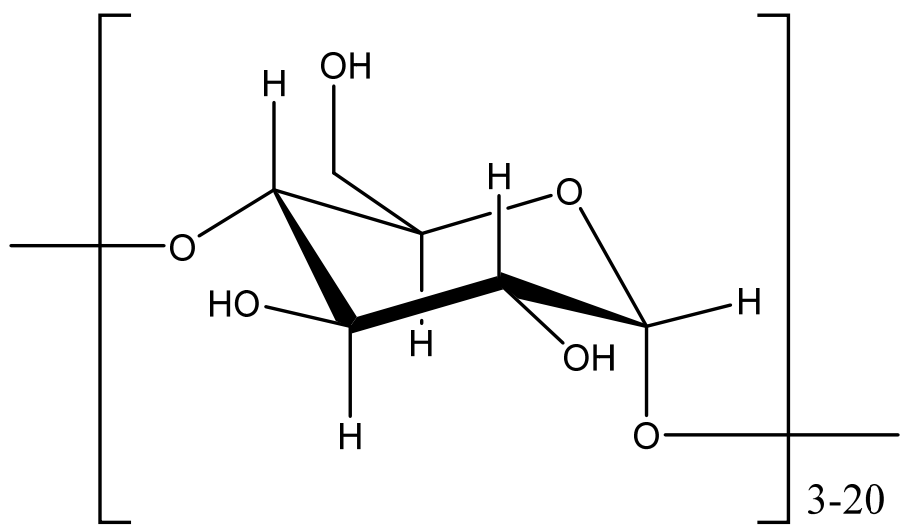

Figure 1. Maltodextrin structure.

Maltodextrins and starches are some of the carbohydrates most commonly employed as vehicles for the microencapsulation of bioactive compounds [9]. Chemically, maltodextrin has different reaction sites in repeating glucose moieties, providing wide alternatives in the chemical conjugation process [5].

The functionalization of MD by incorporating reactive groups to the polymeric network gives these materials specific reactivity against different agents and introduces new properties or enhances properties already present. For that, in this work, a method of functionalization of a matrix-based maltodextrin with urea was developed. The procedure of functionalization gives active amide groups via a short spacer arm. The urea-maltodextrin matrix was synthesized and characterized by chemical techniques.

\section{Materials and Methods}

\subsection{Materials}

The solvents and reagents used during all the work were of analytical reagent grade and used without further purification. Maltodextrin was obtained from a commercial source $(2.85 \%$ of moisture).

\subsection{Derivatization Procedure of Maltodextrin with Urea}

The synthesis of the maltodextrin-urea matrix was carried out by an epoxidationcoupling procedure [10]. In a typical experiment, maltodextrin (25 g) and urea (5 g) were dissolved in $60 \mathrm{~mL}$ of $2 \% \mathrm{NaOH}$ aqueous solution under stirring at room temperature. After a few minutes, epichlorohydrin $(25 \mathrm{~mL})$ and sodium borohydride $(0.03 \mathrm{~g})$ were 
added to the solution. The solution was maintained at room temperature for $3-5 \mathrm{~h}$. The product was washed with distilled water until a negative test of urea. Finally, the solvent was evaporated, and the resulting suspension was centrifugated at 2500 RPM for $15 \mathrm{~min}$. The material obtained after centrifugation was dried in an oven under reduced pressure.

\subsection{Test of Urea}

The assay was carried out by precipitation method, which is based on the formation of urea nitrate with nitric acid [11]. Samples of wash water of the derivatization procedure $(1 \mathrm{~mL})$ were mixed with nitric acid $(0.5 \mathrm{~mL})$; as a result of the presence of urea, a white crystalline precipitate of urea nitrate was formed.

\subsection{Characterization of the Maltodextrin-Urea Matrix}

The maltodextrin-urea material was chemically characterized by FTIR and solubility tests in different solvents. The presence of amine groups on the matrix was evaluated by a test of amines. For the test of amines, a mixture of about $0.05 \mathrm{~g}$ of material and $1 \mathrm{~mL}$ of water was heated until the liquid became turbid. After a few minutes, $1 \mathrm{~mL}$ of $\mathrm{NaOH} 10 \%$ solution and $1 \mathrm{~mL}$ of cupric sulfate $5 \%$ solution were added; the solution acquired a reddish-violet color, verifying the presence of amine groups in the matrix.

The FTIR characterization was performed using IRPrestige-21 (Shimadzu, Japan) with the $\mathrm{KBr}$ sample pellet method, using approximately $1 \%$ of the sample relative to the mass of $\mathrm{KBr}$ per pellet.

Solubility tests were performed by incorporating $0.1 \mathrm{~g}$ of maltodextrin-urea at $10 \mathrm{~mL}$ of the solvent. The solvents used were water, ethanol, 1-butanol, 2-propanol, and ethyl acetate. After mixing the suspension at room temperature, the $\mathrm{pH}$ determination was carried out on a $\mathrm{pH}$ meter.

At the same time, these characterization tests were evaluated with maltodextrin.

\section{Results and Discussion}

\subsection{Functionalization of Maltodextrin with Urea}

Maltodextrin is a saccharide mixture of polymers that consists of D-glucose units. It may be chemically modified to improve its physical and functional characteristics. MD has three hydroxyl groups in every monomeric unit, and they have good potential for derivatization with urea. The functionalization procedure comprises two steps: (a) epoxy functionalization of maltodextrin and (b) coupling of urea to epoxy groups.

Preliminary experiments were carried out by evaluating MD functionalization by two steps: first, the epoxidation of the maltodextrin matrix, and then the coupling of the urea. The epoxy groups were introduced by a known method based on the reaction of hydroxyl groups of maltodextrin with epichlorohydrin in the presence of sodium hydroxide and sodium borohydride [12]. Afterward, the reaction of epoxidated maltodextrin with urea was performed in an aqueous medium. The characterization of these materials shows the instability of epoxy groups.

To simplify the procedures and to obtain the best performance of the procedure, the epoxidation and the urea coupling steps were carried out together (i.e., a "one-pot" functionalization). The synthesis was evaluated during 3,4 , and $5 \mathrm{~h}$ of reaction. In Figure 2 is shown the synthesis pathway of this procedure. The functionalization of maltodextrin added a three-carbon atom arm together with hydroxyl and amide groups. 


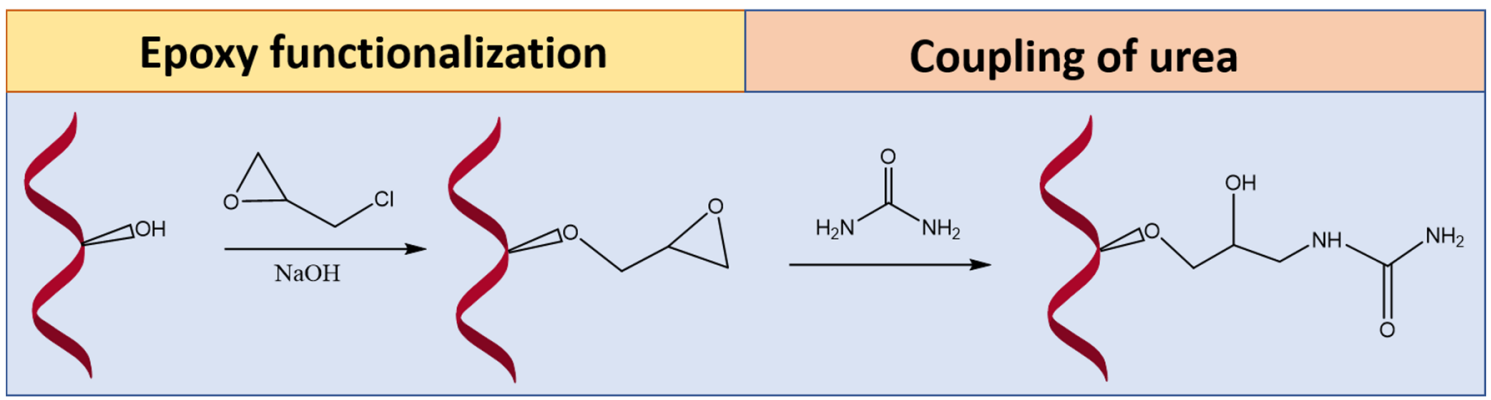

Figure 2. The synthesis pathway of the functionalization of maltodextrin with urea.

\subsection{Characterization of the Materials Synthesized}

In all experiences, the maltodextrin-urea materials obtained showed the same characteristics. These polymers showed a positive amine test and similar FTIR spectra.

From the FTIR spectra (not shown here) of maltodextrin and maltodextrin-urea was identified the functional group present in the structure of these polymers. The FTIR spectrum of maltodextrin showed a characteristic absorption peak at $3431 \mathrm{~cm}^{-1}$ attributed to the presence of $\mathrm{OH}$ groups. Other bands were observed at 2887, 1104, and $1356 \mathrm{~cm}^{-1}$ assigned to stretching vibrations of $\mathrm{C}-\mathrm{H}$ and $\mathrm{C}-\mathrm{O}$ bonds and the symmetric vibrations of C-O-C bonds, respectively $[13,14]$. Compared with maltodextrin, the FTIR spectrum of the maltodextrin-urea matrix shows distinctive absorption bands at around 1650, 1615, and $1470 \mathrm{~cm}^{-1}$, assigned to the stretching vibrations of amide $\mathrm{C}-\mathrm{O}$ bonds, to the bending mode of amide $\mathrm{NH}$ bonds, and to the symmetric stretching vibrations of $\mathrm{C}-\mathrm{N}$ bonds. The presence of these bands indicated the formation of a $\mathrm{C}-\mathrm{NH}-\mathrm{C}=\mathrm{O}$ bond on the maltodextrin-urea polymer $[13,15]$.

On the other hand, the solubility of these materials was evaluated. Maltodextrin was only soluble in water. The maltodextrin-urea (M-D) polymer was soluble in water and ethyl acetate. When the alcoholic solutions of the M-D polymer were added with $0.1 \%$ water, they all showed total solubility. The $\mathrm{pH}$ of the aqueous solution of maltodextrin was 6.5, while in solutions of polymer M-D/solvents, it was 7.0-7.5. These results demonstrate that $\mathrm{OH}$ and amide groups present in the new polymer are of weak basicity, and they are not completely dissociated at $\mathrm{pH}$ above 7.0-7.5.

\section{Conclusions}

In summary, a new maltodextrin-urea matrix was synthesized through a one-pot process. The physicochemical characterization of these materials confirmed the successful functionalization of hydroxide groups free of maltodextrin with urea. Derivatization of maltodextrin with urea confers stability, and terminal amide groups on the surface of this material represent a versatile reactive functional group for conjugation with other molecules for potential applications in the development of new materials.

Author Contributions: V.A.G., M.P. and P.N.: Validation, Formal analysis, Investigation; M.N.K. and C.A.F.: Conceptualization, Methodology, Resources, Writing, Visualization, Supervision, Project administration, Funding acquisition. All authors have read and agreed to the published version of the manuscript.

Institutional Review Board Statement: Not applicable.

Informed Consent Statement: Not applicable.

Data Availability Statement: Not applicable.

Acknowledgments: The authors thank the Capital Semilla (2019); Universidad Nacional del Litoral (UNL), Santa Fe, Argentina; and Universidad Tecnológica Nacional (UTN), San Francisco, Argentina (grant PID 2018 UTN No. 5489), for their financial support of this work.

Conflicts of Interest: The authors declare no conflicts of interest. 


\section{References}

1. Vandghanooni, S.; Eskandani, M. Electrically conductive biomaterials based on natural polysaccharides: Challenges and applications in tissue engineering. Int. J. Biol. Macromol. 2019, 141, 636-662, doi:10.1016/j.ijbiomac.2019.09.020.

2. Rehman, A.; Jafari, S.M.; Tong, Q.; Riaz, T.; Assadpour, E.; Aadil, R.M.; Niazi, S.; Khan, I.M.; Shehzad, Q.; Ali, A.; et al. Drug nanodelivery systems based on natural polysaccharides against different diseases. Adv. Colloid Interface Sci. 2020, 284, 102251, doi:10.1016/j.cis.2020.102251.

3. Yi, Y.; Xu, W.; Wang, H.X.; Huang, F.; Wang, L.M. Natural polysaccharides experience physiochemical and functional changes during preparation: A review. Carbohydr. Polym. 2020, 234, 115896, doi:10.1016/j.carbpol.2020.115896.

4. Liu, J.; Willfo, S.; Xu, C. A review of bioactive plant polysaccharides: Biological activities, functionalization, and biomedical applications. Bioact. Carbohydr. Diet. Fibre 2015, 5, 31-61, doi:10.1016/j.bcdf.2014.12.001.

5. Helal, H.M.; Samy, W.M.; El-Fakharany, E.M.; Kamoun, E.A.; Mortada, S.M.; Sallam, M.A. Maltodextrin- $\alpha$-tocopherol conjugates of vitamin E: Influence of degree of derivatization on physicochemical properties and biological evaluation. J. Drug Deliv. Sci. Technol. 2020, 60, 102097, doi:10.1016/j.jddst.2020.102097.

6. Bonda, A.F.; Regis, L.; Giovannelli, L.; Segale, L. Alginate/maltodextrin and alginate/shellac gum core-shell capsules for the encapsulation of peppermint essential oil. Int. J. Biol. Macromol. 2020, 162, 1293-1302, doi:10.1016/j.ijbiomac.2020.06.194.

7. Du, Q.; Tang, J.; Xu, M.; Lyu, F.; Zhang, J.; Qiu, Y.; Liu, J.; Ding, Y. Whey protein and maltodextrin-stabilized oil-in-water emulsions: Effects of dextrose equivalent. Food Chem. 2021, 339, 128094, doi:10.1016/j.foodchem.2020.128094.

8. Siemons, I.; Politiek, R.G.A.; Boom, R.M.; Van der Sman, R.G.M.; Schutyser, M.A.I. Dextrose equivalence of maltodextrins determines particle morphology development during single sessile droplet drying. Food Res. Int. 2020, 131, 108988, doi:10.1016/j.foodres.2020.108988.

9. Ribeiro, A.M.; Shahgol, M.; Estevinho, B.N.; Rocha, F. Microencapsulation of Vitamin A by spray-drying, using binary and ternary blends of gum arabic, starch and maltodextrin. Food Hydrocoll. 2020, 108, 106029, doi:10.1016/j.foodhyd.2020.106029.

10. Aniulyte, J.B.J.; Bryjak, J. Activation of cellulose-ased carriers with pentaethylenedexamine. Proc. Estonian Acad. Sci. Chem. 2006, $55,61-69$.

11. United States Pharmacopeial Convention, Inc. The United States Pharmacopeia: USP 31; The National Formulary: NF 26; United States Pharmacopeial Convention: Rockville, MD, USA, 2008.

12. Šafař́k, I.; Šafaříkova, M. Black substrate for spectrophotometric determination of cellulose activity in coloured solutions. J. Biochem. Biophys. Methods 1991, 23, 301-306, doi:10.1016/0165-022X(91)90005-H.

13. Silverstein, R.; Bassler, G.C.; Morrill, T.C. Spectrometric Identification of Organic Compounds, 5th ed.; Wiley \& Sons, Inc.: Chichester, UK, 1991; ISBN 0-471-63404-2.

14. Qiu, C.; Qin, Y.; Jiang, S.; Liu, C.; Xiong, L.; Sun, Q. Preparation of active polysaccharide-loaded maltodextrin nanoparticles and their stability as a function of ionic strength and pH. LWT Food Sci. Technol. 2017, 76, 164-171, doi:10.1016/j.1wt.2016.10.053.

15. Chiang, T.C.; Hamdan, S.; Osman, M.S. Urea Formaldehyde Composites Reinforced with Sago Fibres Analysis by FTIR, TGA, and DSC. Adv. Mater. Sci. Eng. 2016, 2016, 5954636, doi:10.1155/2016/5954636. 